\title{
Clinical time and postoperative sensitivity after use of bulk-fill (syringe and capsule) vs. incremental filling composites: a randomized clinical trial
}

\author{
Chane TARDEM(a) \\ Elisa Gomes ALBUQUERQUE(a) \\ Letícia de Souza LOPES(b) \\ Stella Soares MARINS(a) \\ Fernanda Signorelli \\ CALAZANS(a) \\ Luiz Augusto POUBEL(a) \\ Roberta BARCELOS(a) \\ Marcos de Oliveira \\ BARCELEIRO(a) \\ (a) Universidade Federal Fluminense - UFF, \\ Instituto de Saúde de Nova Friburgo, \\ Departamento de Formação Específica, \\ Nova Friburgo, RJ, Brasil. \\ (b) Universidade do Estado do Rio de Janeiro - \\ UERJ, Departamento de Dentística, Rio de \\ Janeiro, RJ, Brasil.
}

Declaration of Interests: The authors certify that they have no commercial or associative interest that represents a conflict of interest in connection with the manuscript.

Corresponding Author:

Marcos Barceleiro.

E-mail: marcosbarceleiro@id.uff.br

https://doi.org/10.1590/1807-3107bor-2019.vol33.0089

Submitted: May 9, 2019

Accepted for publication: July 16, 2019

Last revision: August 16, 2019
Abstract: The objectives of this double-blind randomized clinical trial were to compare (a) the clinical times and (b) the occurrence and severity of postoperative sensitivity, of posterior restoration that used a universal adhesive, in a self-etch or selective enamel-etching technique, along with incremental or bulk-fill composites (presented in syringes or capsules). A total of 295 posterior restorations were placed according to the following groups: SETB - self-etch/bulk-fill in syringe; SETC - self-etch/bulk-fill in capsules; SETI - self-etch/incremental; SEEB - selective enamel-etching/bulk-fill in syringe; SEEC selective enamel-etching/bulk-fill in capsules; and SEEI - selective enamel-etching/incremental. Clinical time was assessed by a reason $\left(\mathrm{s} / \mathrm{mm}^{3}\right)$ between the total volume of resin inserted and the total time required to perform the restorations. Postoperative sensitivity was evaluated using two scales (Numeric Rating Scale and Visual Analogue Scale). Mean clinical time results, analyzed by Wald's Chi-square, showed significant statistical differences among all groups $(p<0.001)$, indicating that the restorative strategy affected the time required for the restoration. A generalized estimating equation model statistical analysis, performed to compare postoperative sensitivity, showed that neither the restorative technique, the adhesive strategy nor the presentation mode of the bulk-fill composite affected the overall risk of postoperative sensitivity (4.06 [2.22-6.81]). The use of bulk-fill composite, presented in capsules or syringes, is less time consuming and does not increase the risk or intensity of postoperative sensitivity relative to the traditional incremental technique.

Keywords: Dental Adhesives; Composite Resins; Randomized Controlled Trial.

\section{Introduction}

The quest to simplify restorative procedures in a way that does not sacrifice quality but rather improves the results of the procedure has persisted, and the continued pursuit of this goal can be observed in the new technological advances that dental material manufacturers release periodically. A good way of illustrating the ongoing nature of this quest 
- Clinical time and postoperative sensitivity after use of bulk-fill (syringe and capsule) vs. incremental filling composites: a randomized clinical trial

can be observed in the research and developments related to dentin adhesive systems. The recent launch of universal adhesive systems and the evaluations performed in laboratory test $\mathrm{s}^{1,2}$ and the first results of clinical evaluation ${ }^{3,4}$ have shown that the practical results of this technological innovation have indeed achieved a simplified form of a restoration procedure that does not sacrifice quality.

As was the case with adhesive systems, manufacturers have undertaken major efforts to modify formulations of composite resins in their organic matrix, in their composition, in their shape and volume of filler particles, and in other components, such as photoinitiators, to improve the final results of the restorations. ${ }^{5}$ Despite all of these advances, composite resins still confront the problem of the stress produced by the polymerization shrinkage, which can cause, among other problems, cusp deflection, ${ }^{6}$ postoperative sensitivity, ${ }^{7}$ marginal discoloration, ${ }^{6}$ and recurrent caries. ${ }^{8}$

As a result, the most popular restorative technique used by dentists seeking to reduce or avoid such problems is the conventional incremental technique. However, in spite of its status as the most popular restorative technique, there are still problems associated with the incremental technique. Perhaps the most important of these relates to the time required to complete a larger volume restoration, as is normally the case with the posterior teeth, ${ }^{9}$ and the potential incorporation of voids within the restoration. ${ }^{10}$ In an effort to resolve these problems, and to continue to simplify and accelerate restorative procedures, the industry has introduced bulk-fill resins. These bulk-fill resins have good mechanical properties ${ }^{11,12}$ even when used in increments of 4 to $5 \mathrm{~mm}$ in thickness, and the first reports of clinical evaluation have been very promising. . $^{3,14}$

Although several authors have studied these two technological innovations, one advantage for both from the clinical point of view is the possibility of performing faster restorative procedures. However, there have not yet been any clinical analyses where bulk-fill has been used in cavities prepared with universal adhesive systems. It is not known, for example, how much time would actually be saved by performing a restoration with these two materials. It is also not known if the presentation mode (capsule or syringe) can influence this outcome, nor is it clear what the immediate clinical outcome would be from the patient's perspective in terms of the experience of postoperative sensitivity.

Thus, one objective of this randomized clinical trial was to evaluate the occurrence and severity of postoperative sensitivity in restorations using a universal adhesive system, with or without selective enamel-etching, and a conventional composite resin inserted by the incremental technique or a bulk-fill composite resin presented in syringes or capsules. Another objective was to compare the difference in the time required to perform the restorations using these six respective techniques (incremental with conventional composite resin or bulk-filling with bulk-fill composite resin, presented in syringes or capsules, in cavities prepared with universal adhesives with or without selective enamel-etching). Considering the objectives, the respective null hypotheses tested were that in posterior restorations with composite, a) the postoperative sensitivity would not be influenced by the restorative materials and techniques, and $b$ ) the mean clinical time to perform the whole restoration using the faster technique (bulk-filling restoring technique) would not be less than the restorations performed using the slower technique (incremental restoring technique).

\section{Methodology}

This was a randomized, double-blind (patient and evaluator) clinical trial performed using the protocol outlined by the Consolidated Standards of Reporting Trials (CONSORT) $)^{15}$ conducted at the School of Dentistry of Fluminense Federal University, from August 17, 2017, to September 30, 2017. This study was approved by the Research Ethics Committee of the local University under protocol number 2.063.508, and it is registered at ClinicalTrial.gov under number NCT03343184. All participants were informed about the study's objectives and the nature of those objectives. Once they consented to participate in the research, a signed voluntary and informed consent form was obtained from each patient prior to initiating the restorative clinical procedures with the materials described in Table 1. 


\section{Selection of patients: sample calculation, inclusion and exclusion criteria and recruitment}

Considering that the null hypothesis tested was related to the risk of postoperative sensitivity, this was the primary outcome defined for this study. Further considering that the nonstimulated postoperative sensitivity in composite resin restorations in posterior teeth in randomized clinical trials averaged $20 \%, 7,16$ 43 cavities were required to generate a $90 \%$ chance of detecting, at a significance level of $5 \%$ and above, a difference of $20 \%$ in the risk of postoperative sensitivity between any of the experimental conditions. However, considering the risk of patient losses intrinsic to randomized clinical trials, the authors chose to increase the number of cavities in each group by $15 \%$. Thus, it was decided to use 53 cavities per group. These calculations were performed on a calculator for Binary outcome superiority trials available at no charge at www.sealedenvelope.com.

The study director performed the initial clinical exams to determine whether patients met the inclusion criteria for this study. These recruited patients appeared for screening, seeking dental treatment at the University, and thus generating a convenience sample. Patients needed to be at least 18 years old, were required to

Table 1. Composition and application mode of materials used in the study.

\begin{tabular}{|c|c|c|}
\hline Materials & Batch $n^{\circ}$ & Composition \\
\hline $\begin{array}{l}\text { Scotchbond } \\
\text { Universal } \\
\text { Adhesive } \\
\text { (3M ESPE, St } \\
\text { Paul, , USA) }\end{array}$ & 601317 & $\begin{array}{l}\text { 1. Etchant: } 34 \% \text { phosphoric acid, water, synthetic } \\
\text { amorphous silica, polyethylene glycol, aluminum oxide. } \\
\text { (Scotchbond Universal Etchant) } \\
\text { 2. Adhesive: MDP phosphate monomer, dimethacrylate } \\
\text { resins, HEMA, methacrylate- modified polyalkenoic } \\
\text { acid copolymer, filler, ethanol, water, initiators, silane }\end{array}$ \\
\hline
\end{tabular}

\section{Filtek One Bulk \\ (3M ESPE)}

Shade A2

\section{2}

(c)

Resin Matrix: AUDMA (urethane aromatic dimethacrylate) / UDMA / 1,12-dodecane-DMA (12-dodecane dimethacrylate);

Fillers: Combination of a non-agglomerated/ non-aggregated $20 \mathrm{~nm}$ silica filler, a non-agglomerated/ non-aggregated 4 to $11 \mathrm{~nm}$ zirconia filler, an aggregated zirconia/silica cluster filler (comprised of $20 \mathrm{~nm}$ silica and 4 to $11 \mathrm{~nm}$ zirconia particles) and a ytterbium trifluoride filler consisting of agglomerate $100 \mathrm{~nm}$ particles; $76.5 \mathrm{w}+\%, 58.4$ vol\%. Photoinitiator: Camphorquinone

Resin Matrix: AUDMA (urethane aromatic dimethacrylate) / UDMA / 1,12-dodecane-DMA (12-dodecane dimethacrylate);

Filtek Bulk Fill Posterior Restorative (3M N693115 ESPE) Shade A2

Filtek Supreme
Ultra
(3M ESPE)
Shade A2

Fillers: Combination of a non-agglomerated/ non-aggregated $20 \mathrm{~nm}$ silica filler, a non-agglomerated/ non-aggregated 4 to $11 \mathrm{~nm}$ zirconia filler, an aggregated zirconia/silica cluster filler (comprised of $20 \mathrm{~nm}$ silica and 4 to $11 \mathrm{~nm}$ zirconia particles) and a ytterbium trifluoride filler consisting of agglomerate $100 \mathrm{~nm}$ particles; $76.5 \mathrm{wt} \%, 58.4$ vol\%. Photoinitiator: Camphorquinone

Resin Matrix: Bis-GMA / Bis-EMA / UDMA / TEG-DMA

Fillers: Combination of a non-agglomerated/ non-aggregated $20 \mathrm{~nm}$ silica filler, a nonagglomerated/ non-aggregated 4 to $11 \mathrm{~nm}$ zirconia

filler, an aggregated zirconia/silica cluster filler (comprised of $20 \mathrm{~nm}$ silica and 4 to $11 \mathrm{~nm}$ zirconia particles); $78.5 \mathrm{w}+\%, 66.3$ vol\%. Photoinitiator: Camphorquinone (polyethylene glycol dimethacrylate, triethylene glycol);
Insert in the cavity bulk increases of up to $5 \mathrm{~mm}$ in thickness, and light- cure each area of the surface of the restoration with a dental curing light appliance light power of $1200 \mathrm{~mW} / \mathrm{cm}^{2}$ for $40 \mathrm{~s}$.
Insert in the cavity bulk increases of up to $5 \mathrm{~mm}$ in thickness, and light- cure each area of the surface of the restoration with a dental curing light appliance light power of $1200 \mathrm{~mW} / \mathrm{cm}^{2}$ for $40 \mathrm{~s}$.
Insert in the cavity oblique increases of up to $2 \mathrm{~mm}$ in thickness, and light- cure each area of the surface of the restoration with a dental curing light appliance light power of $1200 \mathrm{~mW} / \mathrm{cm}^{2}$ for $20 \mathrm{~s}$. 
- Clinical time and postoperative sensitivity after use of bulk-fill (syringe and capsule) vs. incremental filling composites: a randomized clinical trial

demonstrate good general health and acceptable oral hygiene, and had at least 1 posterior tooth with a carious lesion that required restoration, or some deficient posterior restoration in need of replacement (by caries, fracture, or reasons stated by the patient). During patient selection, patients who had teeth to be restored in the same hemi-arch or in different hemi-arches were included. This type of situation (teeth restored in the same hemi-arch) could generate a selection bias; even so, these patients were selected, and the analysis of the results was later corrected for this possible bias.

The lesions had to be Class I or Class II (involving the occlusal surface), of a depth greater than $2 \mathrm{~mm}$, evaluated by means of a bitewing radiograph and ruler, in vital teeth without mobility. In addition, the teeth intended for restorative work needed an antagonist tooth and had to be in occlusion.

Patients with problems requiring endodontic treatment (evaluated by radiography and by a cold vitality test [Roeko-Endo-Frost, Coltène / Whaledent, Langenau, Germany]) were excluded from the study, as well as patients with teeth that had periodontal problems. Pregnant or breastfeeding patients, patients with a known allergy to any of the materials used in the research, and patients currently using or having previously used anti-inflammatories, analgesics, or psychotropic drugs (which could potentially mask the results of postoperative sensitivity) within 15 days of the restorative procedure were also excluded.

\section{Randomization and blinding}

After the screening sessions, 81 patients were selected and 318 teeth were randomized into the six different groups. For randomization, the teeth intended for restoration were treated as independent research subjects and received a code from 1 to 318 . Then, simple randomization was performed in blocks of 6 to ensure an equal number of restorations in each of the six research groups.

The randomization process was performed using a no-charge software available at http://www.sealedenvelope.com. A researcher who was uninvolved in any of the experimental phases performed this procedure. The randomization list was numbered consecutively and individually placed in opaque sealed envelopes. These envelopes were opened on the day of the restorative intervention to prevent disclosure of the randomization scheme. The operator who implemented the interventions was not blinded to the procedure. However, participants and evaluators were blinded to the group allocation during examinations.

\section{Characteristics of cavities and clinical procedures}

After selection of the patients, the study director calibrated four operators already certified as specialists in Restorative Dentistry with at least 5 years of training. The same operators who received this calibration performed the cavity measurements and performed all restorative procedures. Before initiating restorative procedures, all characteristics of each patient (gender and age) and the teeth intended for restoration (tooth type, arch, cavity type, presence of spontaneous sensitivity before restoration, cause of restoration, and number of restored surfaces) were noted (Table 2).

Patients received instructions for oral hygiene and prophylactic care (with pumice and water) before restorative procedures. The operators anesthetized the teeth (Mepisv 3\%, NovaDFL, Rio de Janeiro, Brazil) and performed rubber dam isolation. The cavity design was defined by the removal of carious tissue or defective restorations using a spherical diamond bur (\# 1013; KG Sorensen, Barueri, Brazil) mounted in a high-speed handpiece with air-water spray. After cavity preparation, measurements of width, length, and depth of occlusal and proximal boxes were taken with a periodontal probe (\# 6 Satin Steel Handle, mm, Hu-Friedy, Chicago, USA) and recorded and annotated. In the case of cavities with the involvement of proximal faces, precontoured metal matrices (Unimatrix, TDV, Joinville, Brazil) and proximal wedges were positioned and adapted to obtain the proximal contour of the restorations.

At this moment, the envelopes with the groups were opened, and finally, the operators discovered which of the restorative techniques they would use. At this time, the study director would reset a timer in each box and began counting and observing the time required to perform the restorations. This stopwatch was stopped only after all occlusal adjustments and initial finishing of the restorations. Thus, the time 
Table 2. Characteristics of arches and cavities.

\begin{tabular}{|c|c|c|c|c|c|c|c|}
\hline \multirow{2}{*}{ Variable } & \multicolumn{7}{|c|}{ Group (n) } \\
\hline & SEEB $(n=52)$ & $\operatorname{SETB}(n=52)$ & $\operatorname{SEEC~}(n=47)$ & $\operatorname{SETC}(n=47)$ & SEEI $(n=48)$ & $\operatorname{SETI}(n=49)$ & Total $(n=295)$ \\
\hline \multicolumn{8}{|l|}{ Tooth } \\
\hline Premolar & 19 & 24 & 19 & 30 & 14 & 20 & 126 \\
\hline Molar & 33 & 28 & 28 & 17 & 34 & 29 & 169 \\
\hline \multicolumn{8}{|l|}{ Cavity classification } \\
\hline Class I & 31 & 32 & 24 & 22 & 35 & 32 & 176 \\
\hline Class II - 1 proximal surface & 16 & 13 & 15 & 20 & 10 & 15 & 89 \\
\hline Class II - 2 proximal surfaces & 5 & 7 & 8 & 5 & 3 & 2 & 30 \\
\hline \multicolumn{8}{|l|}{ Restoration depth } \\
\hline $2-3.9 \mathrm{~mm}$ & 31 & 26 & 24 & 30 & 27 & 26 & 164 \\
\hline$\geq 4 \mathrm{~mm}$ & 21 & 26 & 23 & 17 & 21 & 23 & 131 \\
\hline \multicolumn{8}{|l|}{ Caries } \\
\hline Absent & 39 & 41 & 26 & 34 & 42 & 43 & 225 \\
\hline Present & 13 & 11 & 21 & 13 & 6 & 6 & 70 \\
\hline \multicolumn{8}{|l|}{ Restoration reason } \\
\hline Caries & 13 & 11 & 21 & 13 & 6 & 6 & 70 \\
\hline $\begin{array}{l}\text { Restoration change for } \\
\text { aesthetic reasons }\end{array}$ & 29 & 32 & 17 & 20 & 35 & 34 & 167 \\
\hline $\begin{array}{l}\text { Restoration change for } \\
\text { other reasons }\end{array}$ & 10 & 9 & 9 & 14 & 7 & 9 & 58 \\
\hline
\end{tabular}

required for the application of the adhesive system and completion of the entire restorative procedure was recorded individually for each tooth, so that the average time (in seconds) needed to perform each restorative technique could be evaluated per $\mathrm{mm}^{3}$ of composite resin used (width $\times$ length $\times$ depth of each restored occlusal or proximal box). Operators, however, did not know that their procedures were being timed, and they were instructed to perform the restorative procedures as perfectly as they were able, from a technical point of view, without worrying about the relative speed of the restorations.

Restoration procedures were performed according to the following groups:

a. SEEI: Universal adhesive with selective enamel-etching and traditional composite with the incremental technique (Control group)

b. SETI: Universal adhesive without selective enamel-etching and traditional composite with the incremental technique

c. SEEB: Universal adhesive with selective enamel-etching and bulk-fill composite in a syringe with the bulk-filling technique

d. SETB: Universal adhesive without selective enamel-etching and bulk-fill composite in a syringe with the bulk-filling technique

e. SEEC: Universal adhesive with selective enamel-etching and bulk-fill composite in a capsule with the bulk-filling technique

f. SETC: Universal adhesive without selective enamel-etching and bulk-fill composite in a capsule with the bulk-filling technique

Batch numbers and composition of materials used in the study are presented in Table 1. The Scotchbond Universal adhesive system, also known as Single Bond Universal in some countries (3M ESPE, St. Paul, USA), was applied in the self-etching mode, with (SEE) or without (SET) selective enamel-etching with 37\% phosphoric acid (FGM, Joinville, Brazil) according to the manufacturer's instructions (Table 1). The restorations were performed using Filtek Supreme Ultra nanofilled composite resin (3M ESPE, St. Paul, 
- Clinical time and postoperative sensitivity after use of bulk-fill (syringe and capsule) vs. incremental filling composites: a randomized clinical trial

USA), also known as Filtek Z350XT or Filtek Supreme $\mathrm{XTE}$ in some countries, in the groups where the incremental a) technique was used, using the oblique incremental technique, with increments of no more than $2 \mathrm{~mm}$ in thickness, while the Filtek Bulk Fill Posterior Restorative composite resin (b) in syringes (3M ESPE,, St. Paul, USA) or Filtek One Bulk Fill (c) in capsules (3M ESPE,, St. Paul, USA) were used in the groups where the bulk-filling technique was used, with increments of no more than $5 \mathrm{~mm}$ in thickness. The adhesive and restorative procedures are described in Table 1. Photopolymerization of the adhesive system and restorative materials was performed using the Radii Cal (SDI, Victoria, Australia) light unit, with a power of $1200 \mathrm{~mW} / \mathrm{cm}^{2}$, the power of which was evaluated before each individual restoration with a radiometer (Hilux Led Max Curing light meter, First Medica, Greensboro, USA).

Once the restorative procedures were completed, the rubber dam was removed, occlusal adjustments were performed via fine and extra-fine diamond points (KG Sorensen, Barueri, Brazil), and the restorations were polished with a spiral disc designed to finish and polish composites (Sof Lex spiral, 3M ESPE,, St. Paul, USA). In the proximal restorations, the proximal contacts were tested with dental floss and the quality of the cervical adaptation was evaluated by means of interproximal radiography. As required, proximal sanding strips (3M ESPE, St. Paul, USA) were used to perform subsequent adjustments.

\section{Clinical evaluation}

The postoperative sensitivity was evaluated over a period of 7 days by the patient through the use of two scales. A numerical rating scale (NRS) was used with five possibilities for the patient to state how much sensitivity there was in each tooth. In this case, the patient had the option of saying whether the sensitivity was equal to 0 (none), 1 (mild), 2 (moderate), 3 (considerable), or 4 (severe). The patient was also instructed to use a Visual Analogue Scale (VAS), which appeared as a straight line, $100 \mathrm{~mm}$ long, with a zero at one end, indicating no sensitivity, and 100 at the other end, indicating unbearable pain, and to make a mark indicating where their postoperative sensitivity would be located along this spectrum.
The patient received a form for each restored tooth, each of which consisted of the two scales, replicated seven times, and was instructed to note the specific day of each record of the sensitivity. The patient was also asked to indicate whether the sensitivity was spontaneous or stimulated, and in the case of stimulated sensitivity, further asked to indicate the cause of the sensitivity (i.e., chewing, heat, cold, or other stimulus).

\section{Statistical analysis}

The total volume of resin inserted into the different cavities and the total time required to perform all of the restorations within each group was calculated, therefore rendering it possible to determine a mean time per volume of resin for each restorative technique used. The difference between the mean values $\left(\mathrm{s} / \mathrm{mm}^{3}\right)$ obtained was evaluated by Wald's Chi-square test $(\mathrm{p}<0.05)$.

Regarding postoperative sensitivity, all patients who experienced at least one episode of postoperative sensitivity within 7 days were considered to be patients with sensitivity, regardless of intensity. The absolute risk of tooth sensitivity was evaluated using Wald's Chi-square test ( $\alpha=0.05)$. The authors did not calculate the relative risk with the confidence interval for the effect size because there were many cases of multiple restorations performed in the same patient. Considering that a single patient had the potential to receive several restorations, statistical analysis was performed using a generalized estimating equation model (GEE) to avoid the cluster effect.

Comparisons of the number of restorations (\%) that experienced spontaneous postoperative sensitivity during the one-week follow-up reports, according to the characteristics of dental arches and cavities, were evaluated using Wald's Chi-square test $(\alpha=0.05)$. The authors performed comparisons according to the tooth group (molar or premolar), cavity classification and number of surfaces (class I, class II with 1 proximal surface or class II with 2 proximal surfaces), restoration depth (between 2.0 and $3.9 \mathrm{~mm}$ or more than $4.0 \mathrm{~mm}$ ), and caries occurrence before cavity preparation (absent or present). Comparison of the TS intensity (NRS data) of the six groups was not performed because 
of the low frequency of postoperative episodes. The tests were performed using SPSS for Windows software (version 21.0).

\section{Results}

\section{Characteristics of participants and restored cavities}

During the preparation of the cavity, 12 teeth had to be removed from the study because of pulp exposure and 11 teeth had to be removed from the study because the patient refused to continue participating in the clinical trial. Thus, the final number of restorations performed was 295, distributed among the 6 groups as described in Figure, which represents the flow of the patients involved in this study. The restorative protocols were performed exactly as initially planned. Table 2 illustrates the details of the patients and preparations. All participants attended the control visit after 7 days.

\section{Average time to perform restorations}

Table 3 shows the average times required to perform the restorations for each of the six restorative schemes evaluated. The results tested by Wald's Chi-square showed significant differences among all groups $(\mathrm{p}<0.001)$, which means that the restorative strategy influenced the time required for the restoration. It should be noted that the procedure performed in the SEEC group required the shortest mean time per restoration volume $\left(11.03[6.86] \mathrm{s} / \mathrm{mm}^{3}\right)$, followed by the procedure

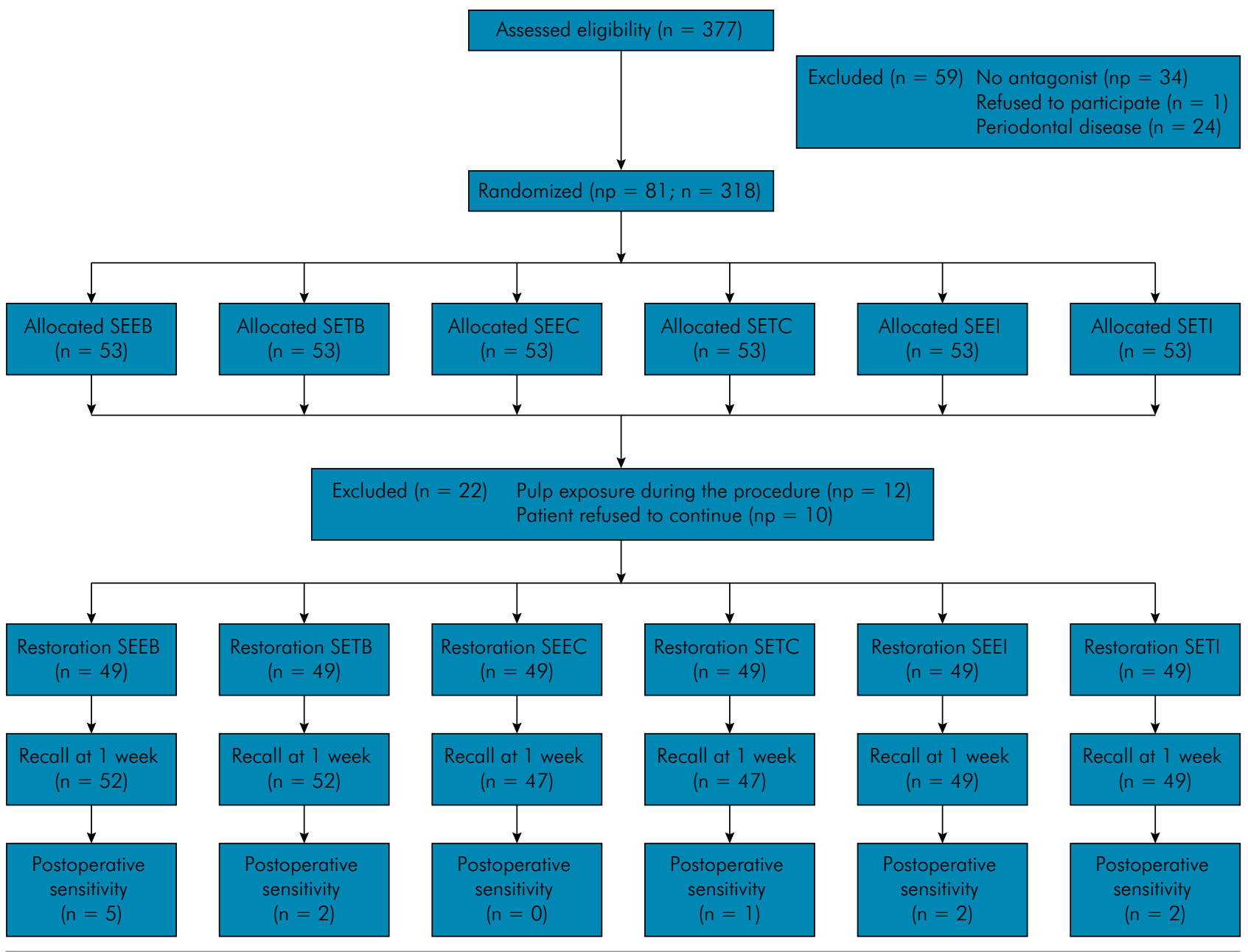

Figure. Participant flow diagram in the different phases of the study design. $\mathrm{Np}=$ number of participants; $\mathrm{N}=$ number of restorations. 
- Clinical time and postoperative sensitivity after use of bulk-fill (syringe and capsule) vs. incremental filling composites: a randomized clinical trial

performed in the SETC group (13.76 [9.18] s/ $\left.\mathrm{mm}^{3}\right)$. The times required in groups SETB (16.04 [17.68] $\left.\mathrm{s} / \mathrm{mm}^{3}\right)$ and SEEB $\left(20.14[22.96] \mathrm{s} / \mathrm{mm}^{3}\right)$ were higher than those for the previous groups. When these four groups were evaluated individually, there were no statistically significant differences among them $(p>0.05)$. However, when the bulk-fill with syringe groups were evaluated against the bulk-fill with capsules groups (not considering the differences in the adhesive strategy), there were statistically significant differences among them $(p=0.003)$. The times in these four groups were significantly lower than the mean time spent in the groups where the incremental technique was used $\left(21.78[20.08] \mathrm{s} / \mathrm{mm}^{3}\right.$ in the SETI group and 28.05 [28.90] s/ $\mathrm{mm}^{3}$ in the SEEI group). Once again, when the bulk groups were evaluated against the incremental groups, there were statistically significant differences among them $(p=0.000)$.

Table 3 shows that, on average, the restorations performed using the incremental technique take 16.10 $\mathrm{s} / \mathrm{mm}^{3}$ longer compared to those performed with bulk-fill in capsules and shows that the restorations performed using bulk-fill in syringes take $6.39 \mathrm{~s} / \mathrm{mm}^{3}$ longer compared to those performed with bulk-fill in capsules. The time/volume ratio for restorations with bulk-fill resins or with incremental resins did not show a statistically significant difference between the two adhesive strategies.

\section{Postoperative sensitivity}

There were only 12 restorations that presented postoperative sensitivity ( 9 mild and 3 moderate) in 6 (7.40\%) different patients, and regardless of the group, in no case did this sensitivity extend beyond a period of 48 hours. In addition, none of the participants had to use any analgesic to control postoperative pain. In 9 out of the 12 reported restorations from which sensitivity resulted, participants reported that their teeth were sensitive only in the instance of mastication. In another 3 restorations, participants reported that their teeth were sensitive to air and cold conditions (Table 4).

Considering the occurrence of postoperative sensitivity, regardless of time and the scale of assessment, the overall risk of postoperative sensitivity was $4.06 \%$ (95\% CI [2.22-6.81]). Neither the restorative technique (incremental vs bulk), the presentation mode (syringe vs capsule) nor the adhesive strategy (etch-and-rinse vs self-etch) affected the risk of postoperative sensitivity (Table 4).

When the characteristics of dental arches and cavities were evaluated, only the restoration depth was statistically significant (Table 5; $\mathrm{p}=0.009$ ). Cavities deeper than $4 \mathrm{~mm}$ demonstrated more postoperative sensitivity relative to shallower cavities. The tooth type, number of surfaces and restoration reason did not exhibit statistically significant differences (Table 5; $\mathrm{p}>0.05$ ).

Table 3. Description of the time/volume ratio (seconds $/ \mathrm{mm}^{3}$ ) to perform the restorative procedures among the experimental groups.

\begin{tabular}{|c|c|c|c|c|c|}
\hline \multirow[b]{2}{*}{ Placement technique } & \multirow[b]{2}{*}{ Adhesive strategy } & \multicolumn{4}{|c|}{ Time/Volume ratio (seconds $/ \mathrm{mm}^{3}$ ) } \\
\hline & & Mean (SD) & Minimum-Maximum & $\begin{array}{c}\text { Difference } \\
\text { compared to SEEC }\end{array}$ & p-value* \\
\hline \multirow{2}{*}{ Incremental filling } & SEEI (selective enamel etching) & $28.05(28.90)$ & $3.26-117.50$ & 17.02 & 0.000 \\
\hline & SETI (self-etching) & $21.78(20.08)$ & $2.04-104.33$ & 10.75 & 0.000 \\
\hline \multirow{2}{*}{ Bulk Fill syringe } & SEEB (selective enamel etching) & $20.14(22.96)$ & $13.36-102.50$ & 9.07 & 0.024 \\
\hline & SETB (self-etching) & $16.04(17.68)$ & $1.67-75.00$ & 5.01 & 0.033 \\
\hline \multirow{2}{*}{ Bulk Fill capsule } & SEEC (selective enamel etching) & $11.03(6.86)$ & $3.91-31.13$ & $0^{* *}$ & --- \\
\hline & SETC (self-etching) & $13.76(9.18)$ & $2.18-44.75$ & 2.73 & 0.073 \\
\hline \multirow{3}{*}{ Overall } & Incremental & $28.42(27.92)$ & $2.04-117.50$ & 16.10 & $<0.000$ \\
\hline & Bulk Syringe & $18.70(20.43)$ & $1.67-102.50$ & 6.39 & 0.003 \\
\hline & Bulk Capsule & $12.32(8.13)$ & $2.18-44.75$ & $0 * * *$ & --- \\
\hline
\end{tabular}

*Wald's Chi-square (comparison between SEEC and other experimental groups); ${ }^{* *}$ Set to zero because this parameter is the comparison between SEEC and other experimental groups; ${ }^{* *}$ Set to zero because this parameter is the comparison between Capsule and other experimental groups. 
Table 4. Frequency distribution of spontaneous postoperative sensitivity occurrence (regardless of time and scale of assessment) after restorative procedures in the experimental groups.

\begin{tabular}{lcccc}
\hline \multirow{2}{*}{ Placement technique } & Adhesive strategy & \multicolumn{3}{c}{ Postoperative tooth sensitivity [n (\%)] } \\
\cline { 2 - 4 } Incremental filling & SEEI (selective enamel etching) & $2(4.1)$ & $46(95.9)$ & $4.17(0.37-14.76)$ \\
& SETI (self-etching) & $2(4.1)$ & $47(95.9)$ & $4.08(0.35-14.49)$ \\
Bulk Fill Syringe & SEEB (selective enamel etching) & $5(9.6)$ & $47(90.4)$ & $9.62(3.75-21.04)$ \\
& SETB (self-etching) & $2(3.8)$ & $50(96.2)$ & $3.85(0.31-13.72)$ \\
Bulk Fill Capsule & SEEC (selective enamel etching) & $0(0.0)$ & $47(100.0)$ & $0.00(0.00-9.02)$ \\
& SETC (self-etching) & $1(2.1)$ & $46(97.9)$ & $2.13(0.00-12.14)$ \\
Resin* & Incremental & $4(4.1)$ & $93(95.9)$ & $4.73(2.14-9.62)$ \\
Overall* & Bulk Fill (Syringe or Capsule) & $8(4.0)$ & $190(96.0)$ & $3.38(1.24-7.88)$ \\
\hline
\end{tabular}

*Wald Chi-square ( $p>0.05)$.

Table 5. Comparison of the number of restorations (\%) that experienced spontaneous postoperative sensitivity during the one-week follow-up according to the characteristics of dental arches and cavities.

\begin{tabular}{|c|c|c|c|c|}
\hline \multirow{3}{*}{ Variable } & \multicolumn{4}{|c|}{ Postoperative sensitivity } \\
\hline & \multicolumn{4}{|c|}{$\mathrm{N}$ (\% within category) } \\
\hline & Absent & Present & Total & $\mathrm{p}$-value \\
\hline \multicolumn{5}{|l|}{ Tooth } \\
\hline Premolar & $124(98.4)$ & $2(1.6)$ & $126(100.0)$ & $0.234^{*}$ \\
\hline Molar & $159(94.1)$ & $10(5.9)$ & 169 (100.0) & \\
\hline \multicolumn{5}{|l|}{ Cavity classification } \\
\hline Class I & $171(97.2)$ & $5(2.8)$ & $176(100.0)$ & $* *$ \\
\hline Class II -1 proximal surface & 83 (93.3) & $6(6.7)$ & $89(100.0)$ & $0.079^{* *}$ \\
\hline Class II - 2 proximal surfaces & $29(96.7)$ & $1(3.3)$ & $30(100.0)$ & $0.890^{* *}$ \\
\hline \multicolumn{5}{|l|}{ Restoration depth } \\
\hline $2-3.9 \mathrm{~mm}$ & $161(98.2)$ & $3(1.8)$ & $164(100.0)$ & 0.009 \\
\hline$\geq 4 \mathrm{~mm}$ & $122(93.1)$ & $9(6.9)$ & $131(100.0)$ & \\
\hline \multicolumn{5}{|l|}{ Caries } \\
\hline Absent & $217(96.4)$ & $8(3.6)$ & $225(100.0)$ & $0.540^{*}$ \\
\hline Present & $66(94.3)$ & $4(5.7)$ & $70(100.0)$ & \\
\hline
\end{tabular}

*Wald Chi-square ( $p>0.05) ;{ }^{* *}$ Comparison between class I and class II (1 or 2 proximal surfaces).

\section{Discussion}

One objective of the present randomized clinical trial was to evaluate the average time required to perform a restoration using resin in posterior teeth using two technological innovations, universal adhesives and bulk-fill composite resins. Although empirically, it seems logical that the restorations made with bulk-fill composite resin would be executed more rapidly, until now, there has only been in vitro evidentiary data to substantiate this conducted on standardized cavities. ${ }^{9}$ Thus, there was no way to determine how much faster it could be to complete whole procedures, including adhesive system application, occlusal adjustment, finishing and polishing in vivo, that were not evaluated in the in vitro study ${ }^{9}$ but were evaluated in this study and that took into consideration the need to calculate the time to perform the whole restoration.

In this study, associated with the use of a universal adhesive (with or without selective enamel etching) and the kind of presentation (syringe or capsule), restoration with a bulk-fill composite took an average time of 18.70 (20.43) seconds per $\mathrm{mm}^{3}$ in the case of 
- Clinical time and postoperative sensitivity after use of bulk-fill (syringe and capsule) vs. incremental filling composites: a randomized clinical trial

syringes or 12.32 (8.13) seconds per $\mathrm{mm}^{3}$ in the case of capsules of bulk-fill resin applied for the completion of the entire procedure, whereas the traditional procedure, performed with traditional composite resin inserted by the incremental technique, associated with a universal adhesive system and applied in the self-etching mode with selective etching of the enamel, took an average time of 28.05 (28.90) seconds per $\mathrm{mm}^{3}$ of traditional composite resin applied. Thus, we rejected the first null hypothesis, since it could be seen that the association of these two new technologies promoted an economic reduction of time close to $60 \%$ of the time required to complete a restoration in resin in a posterior tooth, exactly as desired by the dentists.

It is also interesting to note, as described in the results section, that when the bulk-fill with syringe groups were evaluated against the bulk-fill with capsules groups (not considering the differences in the adhesive strategy), there were statistically significant differences between them $(p=0.003)$, showing that the presentation mode can also influence the time/volume ratio to perform the whole restoration. In this study, there was a reduction of 35\% when capsules were compared to syringes. During the clinical procedures, the four operators noted that the restorations with bulk-fill in capsules were easier to perform, mainly in three important steps, application of the composite in the cavities, finishing and polishing, and this may be a reason for the reduction in time observed in this study. Perhaps other studies comparing the presentation mode should be performed with bulk-fill or traditional composites in order to confirm the findings of this study.

It is important, however, to emphasize that it would be worth taking extra time if the quality and/or durability of the restorations was reduced by employing the innovative methods. From the patient's point of view, for example, a higher postoperative sensitivity would be an undesired outcome, and that is why the authors decided to evaluate this outcome. Analyses of postoperative sensitivity associated with restorations using bulk-fill composite resin in posterior teeth are rare. ${ }^{13,14,16}$ In general, low values of absolute risk of postoperative sensitivity have been observed when these materials are used. However, when the methodologies of these studies are evaluated, it is worth noting that some studies have made use of a flowable bulk-fill composite resin inside the cavity, followed by the use of a conventional resin at the cavosurface angle, which is a distinctive approach to the one used in this study and prevents a fully analogous comparison of the results. Moreover, when these few studies are evaluated, it is possible to observe that none of them were performed using the association described here, that is, bulk-fill resin along with a universal adhesive; this makes it even more difficult to compare our results with the results of other studies represented in the literature. Thus, the closest approach to ours in terms of methodology was the work of Costa et al. ${ }^{16}$ In that study, the authors compared the postoperative sensitivity in restorations performed with a bulk-fill composite resin, inserted incrementally or with the bulk-filling technique, with a self-etching adhesive system or an etch-and-rinse adhesive. In that study, ${ }_{1}^{16}$ the general absolute risk of postoperative sensitivity was $20.3 \%$. That value is not in accordance with the results found in this study and reflects much higher values of absolute risk than those found in this study $(4.06 \%)$.

Considering that the results found here were better than the average values reflected by results that can be found in the literature, ${ }^{7,16}$ the suspicion of authentic improvement in the postoperative sensitivity pattern should be explained by one of the following two hypotheses. In the first hypothesis, the cause for decreased sensitivity could be associated with the adhesive system used. However, a recent systematic review $^{7}$ concluded that the type of adhesive strategy used in bonding procedures in posterior composite resin restorations does not influence the risk and intensity of postoperative sensitivity immediately after the restorative procedure. When that review ${ }^{7}$ is analyzed, it is noted that the adhesive system used in this study was not used in any of the studies examined in that systematic review. However, some of its predecessors with very similar technology, or some self-etch adhesives produced by other manufacturers but also with very similar technology that used the self-etching mode, were included in that review. ${ }^{7}$ In the conclusion of the mentioned systematic review, the authors state that further studies should be conducted 
to determine the extent to which this assertion would also apply to cavities in deep and broad posterior teeth. Thus, considering that in this study there were no differences related to the adhesive technique, this would lead us to imagine that the adhesive system was also not decisive, as described by Costa et al. ${ }^{16}$ When evaluating Table 5, it is possible to observe that factors such as the number of surfaces involved and groups of teeth, as well as other factors noted as causes of the restoration change or even the type of pre-existing restorative material, did not influence the results, responding to the question asked by Reis et al. ${ }^{7}$ In this study, the cavities' depth influenced the risk of postoperative sensitivity, but the postoperative sensitivity relative risk in these deeper cavities was not influenced by the adhesive technique, and this confirms the assertion that the adhesive technique does not influence immediate postoperative sensitivity, even in deep and wide cavities in posterior teeth. ${ }^{5}$

The second hypothesis could hinge on the restorative technique. For this possibility, however, this work would not be a good parameter because when varying the restorative technique, the authors also chose to vary the restorative material and used a conventional resin for the incremental technique and a bulk-fill composite resin for the bulk technique. To say that the incremental technique alone causes more or less sensitivity than the bulk-filling technique would be incorrect, or certainly not sufficiently conclusive, based only on our results. Costa et al., ${ }^{16}$ however, when comparing the two restorative techniques, using the same bulk-fill resin in the two techniques, demonstrated that the overall risk of immediate postoperative sensitivity was not affected by the adhesive strategy (etch-and-rinse/ self-etch) or the filling technique (incremental/bulk). Thus, it seems that the restorative technique should not have influenced the outcomes. However, the restorative materials themselves as used in this study may have had a decisive impact on the results found here relative to the higher values of immediate postoperative sensitivity found in the work of Costa et al. ${ }^{16}$ When the composition of Filtek Bulk Fill (Filtek Bulk Fill Posterior Restorative or Filtek One Bulk Fill) is evaluated, some components, such as an aromatic dimethacrylate (AUDMA), additional fragmentation molecules (AFM), urethane dimethacrylate (UDMA), and 1,12-dodecane dimethacrylate (DDMA), are found in its resin matrix. ${ }^{17}$ It is known that the inclusion of these monomers in the resin matrix allows the polymeric network to relax and reorganize, especially in high-stress regions, providing a potential mechanism for stress relief that enables the network to reorganize and adapt during the polymerization to decrease the shrinkage without developing significant stresses. ${ }^{18}$ Furthermore, these materials contain additional zirconia filler and substitution of glass fillers with zirconia/ silica fillers (2.5 and $5.0 \mathrm{wt} \%$ ), which improves some of their mechanical properties, such as flexural strength and fracture toughness. ${ }^{19}$ Another point that should be evaluated is the fact that the nanocluster particles of the composite resins used in this study possess different mechanical properties relative to filler particles seen in the spherical mixed oxide and isofillers of the composite resins used in Costa et al. ${ }^{16}$ 's study. The shape of bulk-fill fillers used in that study, approaching roundness-of-shape, were shown to positively influence the translucency and to improve the depth of cure ${ }^{19}$ but compromised the mechanical properties relative to the nanohybrid composites. ${ }^{17}$

The presentation mode, in the case of the bulk-fill groups, did not influence the risk or intensity of postoperative sensitivity. As the overall incidence was very low, it was not possible to find significant differences among the groups, but it should be noted that we had only one case (in a total of 94 restorations) of mild postoperative sensitivity that lasted only 24 hours in the capsules groups versus 7 (in a total of 104 restorations) in the syringes groups. Although it was not possible to find statistically significant differences between the presentation modes, these results may show a tendency of lower postoperative sensitivity when using bulk-fill composites in capsules, probably because of the lower risk of the incidence of internal gaps and voids when compared to the syringe presentation mode. The authors believe that further studies should be done to investigate this tendency. 
- Clinical time and postoperative sensitivity after use of bulk-fill (syringe and capsule) vs. incremental filling composites: a randomized clinical trial

However, when the results of this study are evaluated separately, with no comparative considerations to other results in mind, and moreover, when we stop comparing only the overall risk to take stock of the risks group-by-group, we are able to note that the risk in the group where the bulk-fill resin was combined with the adhesive system had values similar to those found when using the conventional resin in incremental mode, which is what was sought after when associating these new technologies. Again, clinical follow-up of these restorations is important in assessing the AFR of these associations, but the early analyses of the immediate results appear very promising.

\section{Conclusions}

Based on the results of this study, the authors concluded that the use of bulk-fill composite, presented in capsules or syringes, is less time consuming and does not increase the risk or intensity of postoperative sensitivity relative to the traditional incremental technique.

\section{Acknowledgments}

This work was supported by the National Council for Scientific and Technological Development (CNPq IC 177759) and Coordination for the Improvement of Higher Level Personnel (CAPES) in Brazil.

\section{References}

1. Muñoz MA, Luque-Martinez I, Malaquias P, Hass V, Reis A, Campanha NH, et al. In vitro longevity of bonding properties of universal adhesives to dentin. Oper Dent. 2015 May-Jun;40(3):282-92. https://doi.org/10.2341/14-055-L

2. Michaud PL, Brown M. Effect of universal adhesive etching modes on bond strength to dual-polymerizing composite resins. J Prosthet Dent. 2018 Apr;119(4):657-62. https://doi.org/10.1016/i.prosdent.2017.06.018

3. Lawson NC, Robles A, Fu CC, Lin CP, Sawlani K, Burgess JO. Two-year clinical trial of a universal adhesive in total-etch and self-etch mode in non-carious cervical lesions. J Dent. 2015 Oct;43(10):1229-34. https://doi.org/10.1016/i.jdent.2015.07.009

4. Loguercio AD, de Paula EA, Hass V, Luque-Martinez I, Reis A, Perdigão J. A new universal simplified adhesive: 36-Month randomized double-blind clinical trial. J Dent. 2015 Sep;43(9):1083-92. https://doi.org/10.1016/i.jdent.2015.07.005

5. Opdam NJ, van de Sande FH, Bronkhorst E, Cenci MS, Bottenberg P, Pallesen U, et al. Longevity of posterior composite restorations: a systematic review and meta-analysis. J Dent Res. 2014 Oct;93(10):943-9. https://doi.org/10.1177/0022034514544217

6. Soares CJ, Faria-E-Silva AL, Rodrigues MP, Vilela AB, Pfeifer CS, Tantbirojn D, et al. Polymerization shrinkage stress of composite resins and resin cements - What do we need to know? Braz Oral Res. 2017 Aug;31 suppl 1:e62. https://doi.org/10.1590/1807-3107bor-2017.vol31.0062

7. Reis A, Dourado Loguercio A, Schroeder M, Luque-Martinez I, Masterson D, Cople Maia L. Does the adhesive strategy influence the postoperative sensitivity in adult patients with posterior resin composite restorations?: A systematic review and meta-analysis. Dent Mater. 2015 Sep;31(9):1052-67. https://doi.org/10.1016/i.dental.2015.06.001

8. Kasraei S, Shokri A, Poorolajal J, Khajeh S, Rahmani H. Comparison of cone-beam computed tomography and intraoral radiography in detection of recurrent caries under composite restorations. Braz Dent J. 2017 Jan-Feb;28(1):85-91. https://doi.org/10.1590/0103-6440201701248

9. Vianna-de-Pinho MG, Rego GF, Vidal ML, Alonso RC, Schneider LF, Cavalcante LM. Clinical time required and internal adaptation in cavities restored with bulk-fill composites. J Contemp Dent Pract. 2017 Dec;18(12):1107-11. https://doi.org/10.5005/ip-journals-10024-2184

10. Soares CJ, Rosatto C, Carvalho VF, Bicalho AA, Henriques J, Faria-E-Silva AL. Radiopacity and porosity of bulk-fill and conventional composite posterior restorations-Digital X-ray analysis. Oper Dent. 2017 Nov/Dec;42(6):616-25. https://doi.org/10.2341/16-146-L

11. Jung JH, Park SH. Comparison of polymerization shrinkage, physical properties, and marginal adaptation of flowable and restorative bulk fill resin-based composites. Oper Dent. 2017 Jul/Aug;42(4):375-86. https://doi.org/10.2341/16-254-L

12. Barceleiro MO, Soares GM, Espindola O, Kahn S, Pola Poiate IA, Sampaio Filho HR. Low-shrinkage composites: an in vitro evaluation of sealing ability after occlusal loading. Gen Dent. 2015 May-Jun;63(3):e36-40.

13. van Dijken JW, Pallesen U. Posterior bulk-filled resin composite restorations: A 5-year randomized controlled clinical study. J Dent. 2016 Aug;51:29-35. https://doi.org/10.1016/i.jdent.2016.05.008

14. Bayraktar Y, Ercan E, Hamidi MM, Çolak H. One-year clinical evaluation of different types of bulk-fill composites. J Investig Clin Dent. 2017 May;8(2):1-9. https://doi.org/10.1111/jicd.12210

15. Schulz KF, Altman DG, Moher D; CONSORT Group. CONSORT 2010 statement: updated guidelines for reporting parallel group randomised trials. Int J Surg. 2011;9(8):672-7. https://doi.org/10.1016/j.ijsu.2011.09.004 
16. Costa T, Rezende M, Sakamoto A, Bittencourt B, Dalzochio P, Loguercio AD, et al. Influence of adhesive type and placement technique on postoperative sensitivity in posterior composite restorations. Oper Dent. 2017 Mar/Apr;42(2):143-54. https://doi.org/10.2341/16-010-C

17. Mandava J, Vegesna DP, Ravi R, Boddeda MR, Uppalapati LV, Ghazanfaruddin MD. Microtensile bond strength of bulk-fill restorative composites to dentin. J Clin Exp Dent. 2017 Aug;9(8):e1023-8. https://doi.org/10.4317/jced.53965

18. Falsafi A, Oxman JD, Tse PH, Ton TT. Longer-term postcure measurement of cuspal deformation induced by dimensional changes in dental materials. Int J Dent. 2015;2015:915071. https://doi.org/10.1155/2015/915071

19. Ikeda I, Otsuki M, Sadr A, Nomura T, Kishikawa R, Tagami J. Effect of filler content of flowable composites on resin-cavity interface. Dent Mater J. 2009 Nov;28(6):679-85. https://doi.org/10.4012/dmj.28.679 